\title{
Activity-Based Therapies
}

\author{
Alexander W. Dromerick, ${ }^{* \dagger}$ Peter S. Lum, ${ }^{\dagger+}$ and Joseph Hidler ${ }^{\dagger \dagger}$ \\ *Departments of Rehabilitation Medicine and Neurology, Georgetown University School of Medicine; ${ }^{\dagger}$ Department of \\ Biomedical Engineering, Catholic University; and ${ }^{\ddagger}$ National Rehabilitation Hospital, Washington, DC
}

\begin{abstract}
Summary: Therapeutic activity is a mainstay of clinical neurorehabilitation, but is typically unstructured and directed at compensation rather than restoration of central nervous system function. Newer activity-based therapies (ABTs) are in early stages of development and testing. The ABTs attempt to restore function via standardized therapeutic activity based on principles of experimental psychology, exercise physiology, and neuroscience. Three of the best developed ABTs are constraintinduced therapy, robotic therapy directed at the hemiplegic arm, and treadmill training techniques aimed at improving gait in persons with stroke and spinal cord injury. These treatments appear effective in improving arm function and gait, but they have not yet been clearly demonstrated to be more effective than equal amounts of traditional techniques. Resistance train-
\end{abstract}

ing is clearly demonstrated to improve strength in persons with stroke and brain injury, and most studies show that it does not increase hypertonia. Clinical trials of ABTs face several methodological challenges. These challenges include defining dosage, standardizing treatment parameters across subjects and within treatment sessions, and determining what constitutes clinically significant treatment effects. The long-term goal is to develop prescriptive ABT, where specific activities are proven to treat specific motor system disorders. Activity-based therapies are not a cure, but are likely to play an important role in future treatment cocktails for stroke and spinal cord injury. Key Words: Rehabilitation, cerebrovascular accident, spinal cord injuries, clinical trials, review.

\section{INTRODUCTION}

This review has two purposes. The first is to introduce readers outside of rehabilitation to the rationales for activity-based therapies (ABTs) and to provide an update on the clinical trial results of ABTs for the restoration of motor system function in persons with stroke or spinal cord injuries (SCI). This review will focus on treating motor impairment, but similar efforts are underway with cognitive and language restoration. The second is to consider how lessons from decades of systematic drug development can be applied to the development and validation of $\mathrm{ABT}$ and ask: How can new rehabilitation strategies be developed more rigorously?

\section{REHABILITATION IS ACTIVITY}

Modern clinical neurorehabilitation is grounded in the premise that activity is beneficial to persons with central

Address correspondence and reprint requests to: Alexander W. Dromerick, M.D., Professor of Rehabilitation Medicine and Neurology, National Rehabilitation Hospital, Georgetown University School of Medicine, 102 Irving Street NW, Washington, DC 20010-2949. Email: alexander.w.dromerick@medstar.net. nervous system injury. The notion of activity having therapeutic properties is so deeply ingrained within the field that even in the absence of direct evidence for the efficacy of most therapeutic activities, clinical trials in which patients are not provided therapeutic activity are generally considered unethical. Even the names of the major clinical disciplines imply activity: the primary rehabilitation medical specialty, physiatry, is the joining of two Greek words: physis for physical phenomena, and iatreia, referring to healing. ${ }^{1}$ Similarly, the therapy disciplines of physical, occupational, speech, recreational, and vocational therapy all have obvious activity implications, often improvised during the treatment session. Billions of dollars are spent each year providing such activities to patients with brain and spinal cord injuries.

However, while the use of $\mathrm{ABT}$ to restore lost function may seem obvious, most of the activity in the rehabilitation clinic is focused on compensation rather than restoration. Current treatment practices ("traditional" therapies) focus on the goals of returning the patient to independence as soon as possible while preventing secondary complications such as contracture and joint deformity. In clinical practice, much of the return to independence is by the use of compensatory strategies, such 
as strengthening the unaffected side to assume more work, learning to dress one-handed, and using devices like canes and braces for mechanical support. Contractures and deformities are prevented by teaching the patient to passively stretch the affected upper extremity (UE) with the unaffected UE and by placing the affected limb in a splint or sling. Thus, in traditional treatments, much of the therapy actually discourages use of the affected body parts. What motor retraining that does take place primarily attempts to reduce spasticity and minimize primitive motor reflexes. ${ }^{2}$

\section{SCIENTIFIC BASIS OF THERAPEUTIC ACTIVITY}

Results from rigorous prospective studies of specific ABTs are only now becoming available, but there is nonetheless a substantial scientific foundation for the idea in studies of normal humans and in animal models of injury. While a detailed review is beyond the scope of this review, the scientific foundation of ABT is drawn from many fields. Principles of educational psychology have been applied in both systematic and ad hoc ways for many decades. Many of the newer "restorative" treatments are more rigorous applications of findings regarding the advantages of various training schedules. For example, there are studies comparing random practice versus blocked practice schedules, ${ }^{3}$ skilled versus unskilled task practice, ${ }^{4,5}$ and those which study the generalizability of training in one task to other untrained tasks. Findings from musculoskeletal physiology have been applied by physical therapists in virtually all rehabilitation diagnoses, where, for example, muscle strengthening protocols are routinely used in many clinical care settings, including those for stroke and SCI (see below). Cardiovascular physiology has gained new currency as an important means to improve mobility by increasing the aerobic capacity to tolerate the increased demands of abnormal gait in persons with motor deficits due to stroke and spinal cord injury (see Macko review; this issue). Data from kinesiology the study of normal movement, and motor system neuroscience, now inform decisions about retraining strategies applied by physical and occupational therapists.

The recent findings in neuroplasticity have also led to the current proliferation of work on ABTs. Functional imaging data have demonstrated changes in patterns of brain activation associated with clinical improvement. While a causal link between recovery and these functional imaging changes has not been clearly established, these changes, at minimum, demonstrate the attempt by the injured brain to access alternative anatomical structures and use secondary and tertiary areas to accomplish motor and language tasks. New understanding of the complexity and fluidity of motor cortex representation have led to the realization that, while there is a relatively fixed anatomic representation of large body segments, such as the arm or leg, the representation of subsegments such as a single joint overlaps considerably with the representation of adjacent structures. ${ }^{6,7}$ Moreover, animal models now demonstrate that the representation of these subsegments can be influenced by training such that shifts take place with the trained segment's representation increasing in size. ${ }^{8,9}$ In humans, acquisition of juggling skills has been associated with thickening of the visual association cortex. ${ }^{10}$ While the actual size of these shifts may be only a few millimeters, the magnitude is considerable on a neuroanatomical scale. These findings open the possibility that shifts from injured to uninjured areas could be prescriptively achieved by systematic training protocols; studies of this strategy are now underway in animal models and in humans.

\section{RELEVANT PARAMETERS OF ABTs}

If ABTs were a pharmaceutical, one early step in development would be to determine specific properties including dose, scheduling of administration, half-life, safety, and so on. Activity-based therapies are therapeutic behaviors, not therapeutic molecules, but some parameters analogous to pharmacological properties have been explored.

\section{Better? Or just more?}

A fundamental question in evaluating ABTs is whether specific activities have unique benefits or whether the reported superiority of a particular ABT is simply related to the amount of time or "dosage" of treatment. Until recently, trials of ABTs used usual and customary care as the control, and usually the control groups receive less treatment.

The absolute magnitude of the effects of conventional therapy in the acute and subacute phases after stroke has not been confirmed because a placebo control is generally considered unethical, and because subjects typically make substantial gains due to spontaneous recovery. ${ }^{11}$ Instead, there has been an effort to determine if conventional therapy has a significant dosage effect, which would provide evidence for increasing the amount of therapy above what is currently provided. Several metaanalyses of controlled studies have found that conventional therapies have a weak, but significant, dosage effect. Two early studies found that greater intensities of conventional therapy result in decreased levels of impairment and disability, ${ }^{12,13}$ with larger effects in the neuromuscular variables than in functional outcomes. ${ }^{12} \mathrm{~A}$ more recent analysis separated outcomes into activities of daily living (ADL), walking speed, and dexterity and found that in ADL, significant dosage effects were present when the treatment was delivered within the first 
six months, while nonsignificant effects were seen in the chronic phase. ${ }^{14}$ They estimated that an additional 16 hours of treatment in the first six months after stroke was needed to obtain significant gains in ADL. Significant dosage effects were also seen in instrumental ADL and walking speed, but not in dexterity.

In addition to these meta-analyses, large retrospective studies also support the conclusion that conventional therapy has a significant dosage effect. In a study of outcomes at 70 skilled nursing facilities, an increased intensity of physical therapy and occupational therapy resulted in increased ADL ability and decreased length of stay. ${ }^{15}$ In two retrospective studies of high and low intensity rehabilitation settings, functional gains were significantly related to therapy intensity and rehabilitation duration, but again effects were considered small. ${ }^{16,17}$ Thus, the evidence for a weak, but significant, dosage effect in conventional therapy provides a rationale for aggressively pursuing ABT development.

\section{Safety of ABT: could activity be harmful?}

Despite the general perception that activity is therapeutic, or at worst not harmful, there are concerns regarding ABT. Few data are available, in part because relatively few rigorous trials are available, and in part because safety of ABT is assumed. The cardiovascular safety issues surrounding treadmill training are reviewed elsewhere in this issue. Schallert et al. ${ }^{18,85}$ raised the possibility that too much activity too soon after injury could increase brain pathology. They found activity-dependent lesion enlargement in a rodent model of stroke recovery. Animals forced to use the hemiparetic forelimb immediately after surgically induced stroke were found to have larger stroke lesions than those not forced to use the forelimb. This effect was blocked by MK-801, suggesting an excitotoxic mechanism. No similar effect has been demonstrated in humans, and one small study of constraint therapy ${ }^{19}$ found no evidence of worse results in subjects who received intense motor training during the inpatient rehabilitation phase of stroke care.

The wrong kind of activity might also be deleterious. Taub et al. ${ }^{21}$ suggested "learned nonuse" could account for improvements from constraint-induced movement. In the model of Taub et al. the patient (or lesioned animal) that has difficulty using the affected side will quickly learn to compensate by using the unaffected side. Because the patient or animal continues to use compensatory strategies, the intrinsic recovery that occurs remains "masked." Forcing the animal or patient to use the impaired UE reinforces the long-term use of the UE in ADLs. Another study by Taub et al. ${ }^{21}$ directly supports this concept of learned nonuse; in this experiment, primates whose unaffected arm was restrained until intrinsic recovery occurred showed full use of the now-recovered limb once restraint was removed. Because a substantial portion of clinical rehabilitation is spent teaching patients compensatory strategies, the possibility that current rehabilitation treatments actually increase learned nonuse cannot be dismissed.

\section{SELECTED MOTOR TRAINING METHODS AND CLINICAL TRIAL RESULTS TO DATE}

\section{Constraint-induced therapy}

Behavioral research with deafferented monkeys led to the development of constraint-induced (CI) therapy for humans with impaired upper limbs after stroke. ${ }^{21}$ The procedures of CI therapy involve promoting use of the more-affected upper extremity for a target of $90 \%$ of waking hours by constraining the less-affected extremity for 2 or 3 consecutive weeks with a padded mitt that prevents use of the hand in ADLs. The patients receive a type of task practice from therapists termed "shaping" for many hours per day, for all weekdays during this period (massed practice).

There have been several reviews that support use of CI therapy in chronic stroke populations. ${ }^{22-24}$ This optimism is based mostly on several uncontrolled studies that found CI therapy was effective for improving movement ability in chronic stroke subjects who met a minimal motor criteria of at least 20 degrees of extension at the wrist and 10 degrees of extension at the finger joints. ${ }^{21,25-28}$ Significant pretreatment to post-treatment gains were reported in laboratory scales of motor impairment and measures of amount of use of the impaired limb outside of the lab. A recent placebo-controlled study showed that the gains from CI therapy are not associated with the amount of therapist attention, and so must be due to the activity from the training. ${ }^{29}$

However, the lack of a large scale randomized clinical trial (RCT) that compares CI therapy with dose-matched traditional therapy has led some to argue that the specific merits of CI therapy are currently inconclusive. ${ }^{30-32}$ Skeptics argue that traditional training methods currently used by therapists may be just as effective if delivered at the same high dose used in CI therapy. A few studies do provide evidence that protocols with high levels of motor activity, such as CI therapy, are superior to equal doses of conventional treatment after stroke. The most comprehensive study was done by van der Lee et al. ${ }^{33}$ and involved 66 chronic stroke subjects. They reported that the CI therapy group performed statistically better than a group of subjects who received an equal dose of neurodevelopmental treatment in terms of an arm movement scale. This result is less impressive than it might be because, using minimal clinically important difference estimates, they determined that clinically relevant gains were present only in patients with sensory disorders and hemineglect. Another recent study ${ }^{34}$ also found advantages in a modified form of CI therapy compared with 
traditional therapies in chronic stroke. Dromerick et al. ${ }^{19}$ performed a study with 23 acute stroke subjects that compared CI therapy with an equal dose of traditional therapy. They found that the CI therapy group outperformed the traditional therapy group on an arm impairment scale, with the largest difference in the pinch subscale. While not explicitly using CI therapy procedures, another study of stroke patients 1 to 5 weeks after stroke reported that additional treatment in the form of repetitive practice of movements and functional activities with a trained assistant was more effective than additional conventional treatment from a physical therapist that focused on teaching techniques and encouraging selfpractice. ${ }^{35}$

\section{Robotic arm training}

There has been growing interest over the last 10 years in robotic devices to assist movement retraining. ${ }^{36}$ Robotic systems may provide a novel sensorimotor experience that enhances recovery of movement compared with equal doses of conventional treatment. In subjects that require physical assistance to complete movement tasks, robots might be a cost-effective alternative to hand-over-hand therapy from a therapist. In the upper limb, this is particularly relevant as attempts at neurorehabilitation are often abandoned early on in favor of compensatory strategies.

The robotic device that has received the most clinical testing is the MIT-MANUS (Massachusetts Institute of Technology, Cambridge, MA), ${ }^{37}$ a two degree-of-freedom robot manipulator that assists shoulder and elbow movement by moving the hand of the patient in the horizontal plane. A clinical trial in subacute stroke subjects (4 to 6 weeks after) found that patients who received 25 hours of treatment with MIT-MANUS had greater gains in motor function than controls who only received a placebo treatment $\left(\right.$ see review ${ }^{38}$ ). Recent trials with chronic stroke subjects have demonstrated that MIT-MANUS training produces significant clinical gains in this population as well. ${ }^{39,40}$ A multisite clinical trial of MIT-MANUS compared with conventional therapy is under way.

Another robotic device that has been highly influential is the assisted rehabilitation and measurement (ARM)Guide. ${ }^{41}$ A motorized linear constraint provides activeassisted reaching movements in different directions. After 8 weeks of training in the ARM-Guide, chronic stroke subjects had functional gains and improvements in reaching kinematics ${ }^{42}$; however, a control group that received a matched amount of unassisted reaching movements had identical gains. This emphasized the fact that highly repetitive active movements have therapeutic value, and the added value of assistance from a robot during active movements remains to be demonstrated.
Clinical trials of the mirror-image motion enabler (MIME) robotic device have shown that robotic devices can have advantages compared with equal doses of conventional therapy. ${ }^{43}$ The MIME device incorporates a 6 degree-of-freedom robot that applies forces to the paretic limb during 3-dimensional movements. Chronic stroke subjects who received 8 weeks of training in the MIME had greater gains in terms of clinical and biomechanical measures of strength and reaching extent than a control group that received an equal dose of conventional therapy. Gains in the robot-trained subjects were due, at least in part, to improved muscle activation patterns. ${ }^{44}$ However, gains in clinical scales were identical between the two groups at the 6-month follow-up. A follow-up study in subacute stroke subjects confirmed these results. Arm impairment scores at post-treatment were in favor of the robot-trained group, but both groups were equivalent at the 6-month follow-up (Lum, in press).

\section{Strength training}

Strength training (maximal effort, resistance exercise) has benefits at both muscular and neural levels. Increased torque output within the first month of strength training in deconditioned limbs is predominantly due to neural adaptation and not muscle hypertrophy. Neural adaptation has been reported after strength training in patients with all types of neuromuscular disorders. ${ }^{45,46}$ While the mechanisms that underlie neural adaptation are not fully understood, the result is increased force output accompanied by increased amplitude of surface electromyogram. ${ }^{47}$ After approximately 4 weeks of training, muscular adaptations produce additional gains in peak torque.

Despite the prevalence of weakness after stroke and the potential for positive adaptations at both muscular and neural levels, the belief that excessive effort exacerbates hypertonia has prevented widespread use of strength training in persons with poststroke hemiparesis. Several studies have challenged this belief and found that significant gains in strength are possible without increased hypertonia. ${ }^{48,49}$ However, functional gains were generally smaller or nonexistent. While it is clear that some muscle groups are responsive to strengthening protocols, promoting clinically relevant functional outcomes may hinge on whether strengthening is effective at increasing torque output in joint actions that are profoundly weak after stroke (i.e., elbow extension, finger extension, knee flexion, etc.). If so, it may serve as an effective method for reducing the strength imbalances between agonist and antagonist muscle groups that are common after stroke. ${ }^{50}$ Another relevant factor that may limit the effectiveness of strengthening protocols is task specificity, the concept that gains are greatest in activities that are trained, with reduced effects in similar, but untrained tasks. ${ }^{51}$ For example, previous studies have re- 
ported that isokinetic training produces large gains in isokinetic strength, but smaller gains in isometric strength. ${ }^{52}$ Thus, interventions that combine strengthening with task practice are likely to be the most effective use of strengthening protocols. The strengthening component serves to promote muscular and neural adaptations to decrease the weakness impairment and the task practice promotes transfer of this enhanced ability to performance of functional tasks.

\section{Treadmill training for gait}

First reported by Barbeau et al., ${ }^{53}$ treadmill training in humans is a direct byproduct of the extensive research in animals evaluating activity-dependent neural plasticity in the spinal cord. ${ }^{54}$ After locomotor treadmill training, spinalized cats are able to walk on their hindlimbs with near normal gait patterns at different speeds, while maintaining their full body weight. ${ }^{5,56}$ Without repeated locomotor training sessions, recovery of locomotor ability is significantly compromised. ${ }^{57,58}$ These studies demonstrate that within the rodent and feline spinal cord there exist neuronal circuits capable of producing patterned movements and exhibiting a large degree of neural plasticity that is trainable with appropriate afferent inputs. ${ }^{59}$ In the absence of afferent activity, locomotor patterns are less precise, less coordinated, and weaker. ${ }^{60}$ In fact, animals trained only to stand do so well, but either do not step or step with patterns inferior to untrained animals, ${ }^{61}$ thus strengthening the argument for task-specific therapies. As spinal "learning" is thought to be important in facilitating the return of motor function after spinal cord injury, effective therapeutic interventions must optimally stimulate the necessary afferent inputs needed to train spinal circuits responsible for producing desired rhythmic motor patterns such as walking.

One such intervention that has been touted as "appropriate" for improving walking ability in individuals with neurological injuries such as stroke and SCI is manualassisted body-weight-supported treadmill training. ${ }^{62}$ In this intervention, SCI patients are suspended in a sling in the upright position over a motorized treadmill; therapists manually assist the patient in moving the legs in a gait-like pattern. It is postulated that treadmill training in humans provides a rich sensory environment that promotes spinal plasticity such that central circuits are more receptive to sensory modalities during stepping; therefore, the spared descending drive has a more pronounced effect on eliciting motor output. Early studies evaluated the effectiveness of manually assisted body-weight-supported treadmill training in both individuals with spinal cord injuries ${ }^{63-65}$ and in stroke survivors, ${ }^{66-68}$ reporting that this treatment was more effective than conventional gait training in improving a number of important gait metrics. These included increases in overground walking speed, ${ }^{67,68}$ more natural electromyogram activation patterns, ${ }^{65,68}$ and higher functional walking ability. ${ }^{64}$

Missing from all of these studies was a phase III RCT. Most of these reports were based on a limited subject sample that was not adequately screened or randomized into particular training modalities. In response, Dobkin et al. ${ }^{69}$ launched a large RCT across 6 rehabilitation centers that sought to determine whether this training modality would lead to higher gains in walking ability and lower extremity function than overground gait training. After training 146 individuals with incomplete spinal cord injury, Dobkin et al. reported that gains in walking ability, assessed by overground walking speed in ambulatory subjects, and lower extremity function, assessed using the locomotor portion of the Functional Independence Measure (Dodds et al. ${ }^{89}$ ) for nonambulatory, were no different between treatment groups. $^{70}$ Most subjects demonstrated improvements in walking ability and lower extremity function, gains that exceeded expectations; however, the gains in treadmill-trained subjects were not statistically different than the conventional group.

In summary, the studies reviewed above demonstrate effectiveness of these ABTs in improving aspects of motor performance in patients with stroke and SCI. This confirms the underlying doctrine of rehabilitation, namely that activity is therapeutic. What remains to be determined is whether the tested protocols were optimized, and whether these newer ABTs are superior to equal doses of the more traditional, improvised clinical treatments currently delivered to these patient populations.

\section{APPLYING THE PRINCIPLES OF DRUG DEVELOPMENT TO ABT}

We submit that developing a drug and an $\mathrm{ABT}$ are fundamentally similar processes, modified only slightly for the properties of ABT. The development and testing of therapeutic interventions are most fully developed for pharmaceuticals, and developers of ABTs might hasten progress by systematically applying these methods to rehabilitation treatments. In particular, most ABTs at this time are tangled up in premature phase III and IV evaluation; our discussion will focus on key aspects of phase II development of ABTs.

TABLE 1 outlines the phases of drug development; this model is used by pharmaceutical regulatory agencies around the world. ${ }^{71}$ While the parallels for ABTs are obvious, there are some differences between the development of a therapeutic molecule and a therapeutic behavior. First, "preclinical" development may take place in vitro or in animal models, but many ABTs emerge from the sports training or experimental psychology world and the "preclinical" studies may occur in healthy humans. Second, while manufactur- 
TABLE 1. Development Scheme for Pharmaceuticals and Activity-Based Therapies

\begin{tabular}{|c|c|c|}
\hline $\begin{array}{l}\text { Development } \\
\text { Phase }\end{array}$ & Primary Activity & Goal \\
\hline Discovery & $\begin{array}{l}\text { Discovering changes in motor } \\
\text { system properties driven by a } \\
\text { specific activity }\end{array}$ & $\begin{array}{l}\text {-Identify effects at molecular, cellular } \\
\text { or organ level, or characterization } \\
\text { of effects in normals }\end{array}$ \\
\hline Preclinical & Testing in animal models & $\begin{array}{l}\text {-Confirmation of therapeutic effect in } \\
\text { lesioned animal or healthy human } \\
\text { models }\end{array}$ \\
\hline Phase I & $\begin{array}{l}\text { Testing in healthy volunteers, or in } \\
\text { selected patient population }\end{array}$ & $\begin{array}{l}\text {-Proof of principle, feasibility } \\
\text {-Dose ranging }\end{array}$ \\
\hline Phase II & $\begin{array}{l}\text { Testing in target patient population } \\
\text { - Multiple smaller studies } \\
\text { - Controls: dosage, social } \\
\text { interaction, other }\end{array}$ & $\begin{array}{l}\text {-Preliminary efficacy data } \\
\text {-Refinement of treatment parameters } \\
\text {-Refinement of inclusion-exclusion } \\
\text { criteria } \\
\text {-Estimation of optimal dose } \\
\text {-Evaluation of potential endpoints } \\
\text {-Sample size estimation } \\
\text {-Safety determination } \\
\text {-Development of treatment protocol, } \\
\text { case report form }\end{array}$ \\
\hline Phase III & Testing in population of interest & $\begin{array}{l}\text {-Efficacy determination } \\
\text {-Safety determination } \\
\text {-Economic evaluation }\end{array}$ \\
\hline Phase IV & Postimplementation evaluation & $\begin{array}{l}\text {-Evaluation of implementation outside } \\
\text { study centers } \\
\text {-Safety surveillance }\end{array}$ \\
\hline
\end{tabular}

ing a reproducible and pure drug molecule is usually reasonably straightforward, the "manufacturing" process of an ABT is more complicated. The ABTs are, by their nature, a structured interaction between an injured patient, a therapist, and any device involved in the treatment. Defining and standardizing this interaction between 2 human beings in a reproducible way is a key development step, and a necessary condition for rigorous testing. This definition and standardization is manifested in a treatment protocol that outlines the activities that constitute the ABT, the criteria for advancing in difficulty or duration, and the modifiable parameters available to the therapist. Thus, the underlying "product" being tested is the treatment protocol itself, and despite the plethora of small scale treatment studies published, few include a description of the experimental intervention that allows the reader to determine how reproducible that intervention might be. Finally, regulatory barriers to new drug introduction are quite high in developed countries. In contrast, there are no barriers, other than cost, to the introduction of new ABTs into clinical practice, regardless of supporting data. This unstructured practice complicates the definition of "standard treatment" control in the phase II and III trial setting, and potentially subjects neurologically impaired individuals to interventions which could be ineffective or even harmful.

\section{PRECLINICAL DEVELOPMENT OF ABT}

\section{Targeting "changeable" parameters}

In many management models, it is often constructive to generate a list of factors that may impact the desired goal, and then determine the ability of the organization to change each factor. A similar methodology can be useful in activity-dependent therapies. For example, in FIG. 1., we list a number of factors that may influence a patient's recovery. These range from an individual's age and motivation to the intensity of the intervention. If we plot these factors against our ability to change them, we can target those that lie in the upper right quadrant. Here these factors have a significant impact on an individual's recovery, while at the same time we can strongly influence them. For example, lesion location, age, and cognitive state may be very influential on a patient's recovery yet it is difficult, if not impossible, for clinicians to change them. Conversely, time after injury and the type of intervention may also impact the magnitude of motor recovery and are controllable within the neurorehabilitation setting. It is these factors that should be the target of RCTs as well as clinical interventions.

\section{Animal models}

Animal models are a mainstay of biomedical research, but few rehabilitation treatments undergo animal testing; constraint therapy ${ }^{72,73}$ and treadmill training ${ }^{74}$ being no- 


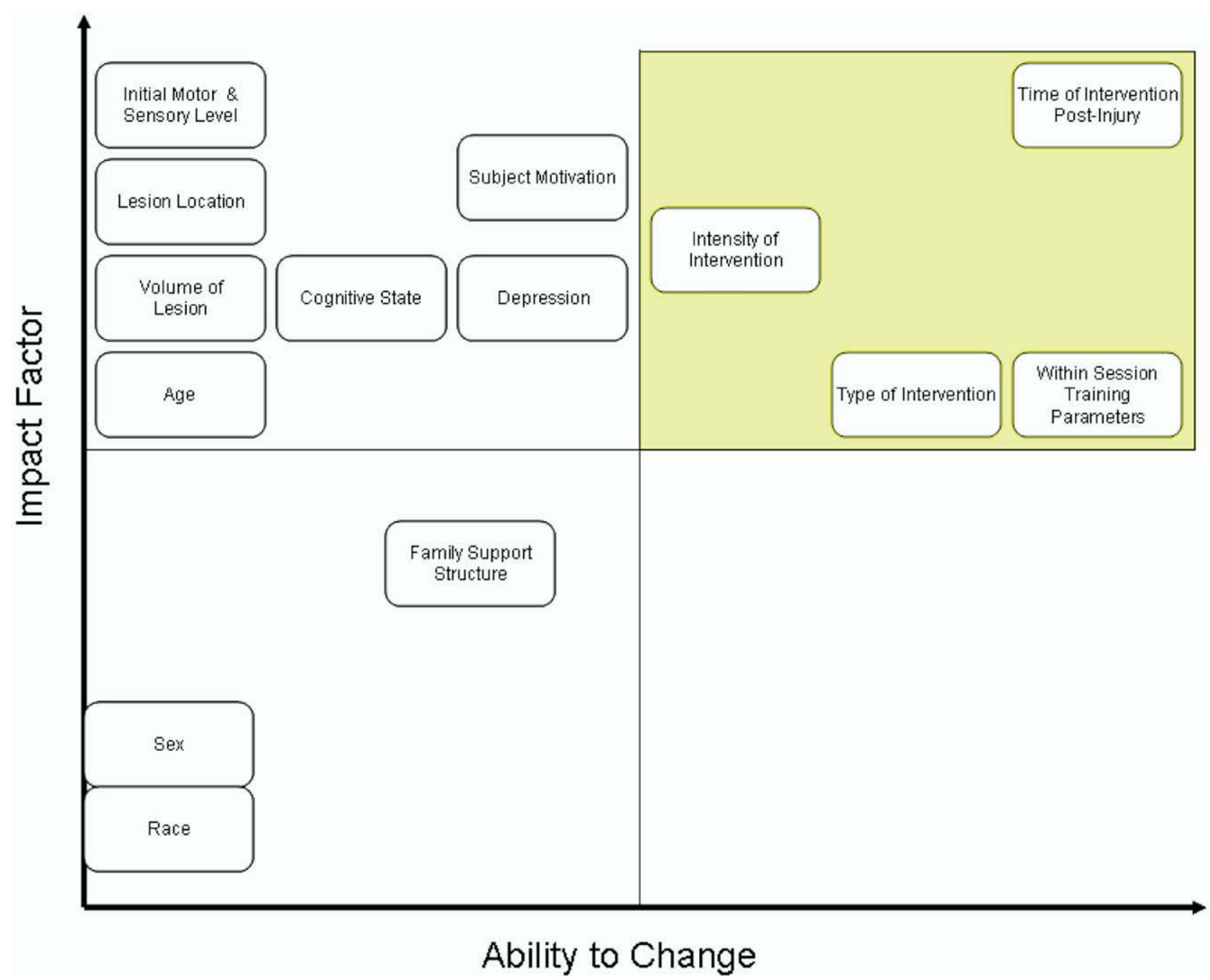

FIG. 1. Factors that may impact an individual's recovery and our ability to change them. Plotting these factors in quadrants allows us to target those factors that have a high impact while at the same time are "changeable" (yellow quadrant).

table exceptions. Relevant subject treatment parameters (see below) can be quickly evaluated in animals at relatively low cost before moving into more complex phase II human studies, hastening the rational development of clinical treatments. But results in animals are not always replicable in human patients. Particularly relevant is the recent experience in acute stroke, where results of neuroprotectant studies in animal models do not always predict phase III results. ${ }^{75}$ Activity-based therapies directed at the motor system face two specific challenges in animal modeling: the differences between human bipedal locomotion and rodent quadripedal locomotion, ${ }^{76}$ and the differences in stroke lesion locations between humans and animal models of stroke. ${ }^{77}$

\section{PHASE I/II DEVELOPMENT OF ABT}

The topic of phase II development of ABT is worthy of extensive examination, and we will highlight only a few of the aspects outlined in TABLE 2. Before launching into a large-scale study to test the effectiveness of any intervention focused on treating motor impairments, a number of important intervention factors need to be considered.

\section{Refinement of dosage}

Dosage in a pharmaceutical trial is usually defined as the number of molecules administered. Defining dosage in the context of a motor ABT is more complex. It could be measured by the amount of time spent in the therapeutic activity, the number of repetitions during treatment, the difficulty of activity, or the intensity (number of repetitions per unit time). In practice, most investigators choose some duration-based ABT delivery schedule that either resembles typical clinical treatment in their home institution or is based on some arbitrary number of treatment sessions or time period. Dose-response curves are rarely if ever 
TABLE 2. Phase II Evaluation of Activity-Based Therapies

\begin{tabular}{|c|c|}
\hline Factor to Be Evaluated & Comments \\
\hline Preliminary efficacy data & $\begin{array}{l}\text {-Often uses surrogate endpoints (strength, coordination, } \\
\text { electrophysiological measure, etc.) }\end{array}$ \\
\hline Refinement of treatment parameters & $\begin{array}{l}\text {-Timing of administration after injury } \\
\text {-Dosing schedule } \\
\text {-Treatment-specific factors: task properties, gait speed, etc. }\end{array}$ \\
\hline Controls & -Consider placebo, Hawthorne, socialization, and dosage effects \\
\hline Inclusion-exclusion criteria & $\begin{array}{l}\text {-Consider neurological impairment, lesion characteristics, demographics, } \\
\text { cognitive impairment, medications, comorbidities }\end{array}$ \\
\hline Dosing & -Testing of multiple doses provides a dose-response curve \\
\hline Evaluation of candidate endpoints for phase III & $\begin{array}{l}\text {-Choice of clinically significant endpoint } \\
\text {-Framed by a "number need to treat" analysis }\end{array}$ \\
\hline Sample size estimation for phase III & $\begin{array}{l}\text {-Dependent on properties of candidate endpoints } \\
\text {-Accuracy of estimate improves with Phase II sample size }\end{array}$ \\
\hline Safety evaluation & $\begin{array}{l}\text {-Presumed low risk for most activity-based therapies, but rarely } \\
\text { systematically collected }\end{array}$ \\
\hline Development of treatment protocol & $\begin{array}{l}\text {-Definable and concrete } \\
\text {-Replicable, so that different clinicians treating same subject would } \\
\text { provide similar treatment } \\
\text {-Distinguishable from control or other treatment }\end{array}$ \\
\hline
\end{tabular}

generated; at most, a second higher or lower dosage is attempted. $^{78}$

One possible solution to determining the correct dosage of an intervention is to continue to train subject populations in a pilot study until the primary outcome measure of interest plateaus for a predetermined amount of consecutive sessions; for example, many locomotion studies use overground walking speed as the primary outcome measure for ambulatory subjects. ${ }^{68,69}$ For such studies, prior to each training session, the subject's gait speed could be measured and if the change in speed does not vary by some noise interval over 6 to 8 sessions, the training stops. Furthermore, rather than basing individual training sessions on time, perhaps it would be best to schedule based on intervention volume. For upper limb studies, this may include the number of attempted (or successful) reaches toward a target. For lower limb studies, it may be the number of steps the individual takes. Each metric would have to be normalized, perhaps by time, which would account for intensity because walking 1500 steps in one hour is significantly different than 1500 steps in 15 minutes!

\section{Refinement of training parameters}

Training parameters are distinct from dosage, as defined above, and consist of the conditions under which the intervention is delivered within a particular treatment session. It is generally assumed that training parameters may significantly influence motor recovery, but they are often selected through experience and heuristic rules. While clinical experience can be helpful in phase I, a critical product of phase II development is quantitative evidence demonstrating how training should be initiated and progressed. The recent publication of a case report using a functional electrical stimulation (FES) cycling paradigm in a celebrity with $\mathrm{SCI}^{79}$ is a case in point, because it has led to widespread application of the technique in SCI. Few studies have investigated FES pedaling characteristics such as stimulation intensity (e.g., stimulation frequency and amplitude), or turnover rate (e.g., cadence), and the number of interacting factors complicates analysis. For example, a flywheel can be used to help maintain pedaling momentum and relieve some of the subject's propulsion requirements throughout the cycle. Thus, once a steady pedaling rate is achieved, there is a continuous interaction between active subject pedaling and crank momentum. So pedaling at a higher cadence may be an illusion that the subject is being trained at a higher workload. Similar misinterpretations are often reported in locomotor studies where it has been suggested that training subjects at faster speeds reduces the amount of assistance trainers must provide apply to the subject's legs and therefore the individual must be stepping better. While the subjects may appear superficially improved, they may simply be taking advantage of momentum rather than truly producing stable stepping patterns under voluntary control.

More preclinical and phase I work would hasten and rationalize development. For example, in the case of FES cycling, evaluating the amount of active work produced during the crank cycle can be compared with healthy individuals so that parameters such as stimulus frequency, timing, and amplitude parameters could be established. These parameters could then be configured so that an SCI subject generates active propulsion at the proper phases of the crank cycle. Similar procedures can be used in locomotion studies. For example, muscle ac- 
tivation patterns can be compared with normative patterns in order to determine the training conditions (e.g. walking speed, level of body-weight support, etc.) under which the subject activates their lower extremity muscles appropriate to the gait cycle. ${ }^{80}$ The idea is to understand how training parameters should be established and then progressed so that the resulting motor pattern(s) are appropriate to the task.

\section{Timing of administration}

It is difficult to envision how to determine the optimal time after injury for a particular intervention other than through direct experimentation. Gresham ${ }^{81}$ referred to timing of treatment as "perhaps the most urgent methodological issue that (rehabilitation) investigators must resolve." Remarkably few studies in the literature, either animal or human, directly address this question in anything but a descriptive fashion. Most of these studies use activity of daily living function as their endpoint rather than the underlying impairments that drive disability.

The assumption in the field has been that earlier is better, ${ }^{82}$ and most case series and a meta-analysis ${ }^{83}$ show a correlation between early intervention and overall outcome. These studies are limited both by their correlative methods and by defining "early" as whenever the patient actually started rehabilitation. One exception is Paolucci et al. ${ }^{84}$ who randomized 145 stroke patients to receive inpatient stroke rehabilitation at less than 20, 20 to 40, or 40 to 60 days after stroke onset. Those who were randomized to the earliest group showed the largest Barthel Index response to treatment upon discharge. A study of $\mathrm{CI}$ therapy during inpatient rehabilitation ${ }^{19}$ found a considerably larger effect size than a similar study ${ }^{33}$ done in chronic patients. Animal models are of uncertain relevance to humans, but they generally support early treatment. The early recovery events in the brains of rodents and primates suggest a period of particular receptivity to experience-dependent mechanisms soon after injury, including synaptogenesis and neurogenesis. ${ }^{85-87}$ But investigators must either systematically determine the best time window, or make their best guess.

\section{Refinement of inclusion-exclusion criteria}

Another important goal of phase II development is refining the target population so as not to include participants in a phase III study who cannot respond to the intervention, yielding a falsely negative result. Even seemingly minor decisions about inclusion and exclusion criteria can profoundly affect the characteristics of subjects enrolled in a study, and these characteristics may determine whether or not an intervention has an effect. We have already mentioned two good examples in ABT, one involving treadmill training, and the other CI therapy. In an important study of treadmill training ${ }^{68}$ it was found that, in general, stroke survivors that received partial body-weight-supported treadmill training demon- strated significantly higher gains in walking ability than those who did not receive body-weight support. A subsequent secondary analysis, ${ }^{88}$ found that only in the most severely impaired subjects were the differences in training modalities significantly different. Similarly, in the study of CI therapy by van der Lee et al., ${ }^{33}$ the positive effect of CI therapy was found primarily in those subjects with hemisensory loss or neglect, and not in those with pure motor impairments. Both studies would have been negative had inclusion criteria excluded these subpopulations, and might have been more strikingly positive had nonresponding classes of patients not been randomized.

\section{CONCLUDING COMMENTS}

As rehabilitation practice becomes more evidence based, and as ABT trial methodology improves, a few points become apparent. First, it is clear that therapeutic activity will retain an important role in rehabilitation for quite some time. Whether theory-driven and well-defined ABT protocols are an improvement over the clinical judgment of experienced clinicians remains to be determined.

Second is the maximal effect size of any ABT: what treatment response can be reasonably expected of $\mathrm{ABT}$ interventions? In the absence of directly relevant data perhaps the best answer lies in the exercise physiology world, where different training paradigms and schedule are applied to elite athletes with the goal of improving their performance. While on a relative scale, better training techniques can give an important advantage over other elite competitors; on an absolute scale of speed or strength most changes are small in magnitude. The target clinical populations for ABT interventions are not elite athletes and often have injuries to the neural mechanisms necessary for new learning. Whether this increases or reduces the potential response to $\mathrm{ABT}$ interventions is not clear, but the results in treadmill training ${ }^{88}$ and CI therapy ${ }^{33}$ suggests that treatment response can vary across different subpopulations.

Finally, another looming question is the role of ABT interventions within a "cocktail" of therapies. There is much discussion that transplanted stem cells may need activity to help target axonal growth and synaptic connections. Phase I/II trials of recovery agents such as growth factors, transcranial magnetic or direct current stimulation, and amphetamine include standardized ABT protocols. These are provided in part to minimize heterogeneity in treatment between subjects, but typically constitute part of the experimental intervention. These combinatorial treatments will complicate clinical trial design and execution, but may offer new therapeutic choices for those with stroke and spinal cord injury. 


\section{REFERENCES}

1. The history of physiatry (online). Available at http://www.physiatry. org/field/index.html. Accessed Date: May 1, 2006.

2. Roth EJ. The elderly stroke patient: principles and practices of rehabilitation management. Top Geriatr Rehabil 1988;3:27-61.

3. Cauraugh JH, Kim SB. Stroke motor recovery: active neuromuscular stimulation and repetitive practice schedules. J Neurol Neurosurg Psychiatry 2003;74:1562-1566.

4. Thielman GT, Dean CM, Gentile AM. Rehabilitation of reaching after stroke: task-related training versus progressive resistive exercise. Arch Phys Med Rehabil 2004;85:1613-1618.

5. Winstein CJ, Rose DK, Tan SM, Lewthwaite R, Chui HC, Azen SP. A randomized controlled comparison of upper-extremity rehabilitation strategies in acute stroke: a pilot study of immediate and long-term outcomes. Arch Phys Med Rehabil 2004;85:620-628.

6. Schieber MH, Poliakov AV. Partial inactivation of the primary motor cortex hand area: effects on individuated finger movements. J Neurosci 1998;18:9038-9054.

7. Nudo RJ, Wise BM, SiFuentes F, Milliken GW. Neural substracts for the effects of rehabilitative training on motor recovery after ischemic infarct. Science 1996;272:1791-1794.

8. Nudo RJ, Milliken GW, Jenkins WN, Merzenich MM. Use-dependent alterations of movement representations in primary motor cortex of adult squirrel monkeys. J Neurosci 1996;16:785-807.

9. Kleim JA, Swain RA, Armstrong KA, Napper RMA, Jones TA, Greenough WT. Selective synaptic plasticity within the cerebellar cortex following complex motor skill learning. Neurobiol Learn Mem 1998;69:274-289.

10. Draganski B, Gaser C, Busch V, Schuierer G, Bogdahn U, May A. Neuroplasticity changes in grey matter induced by training. Nature 2004;427:311-312.

11. Jorgensen HS, Nakayama H, Raaschou HO, Vive-Larsen J, Stoier M, Olsen TS. Outcome and time course of recovery in stroke. Part I: outcome. The Copenhagen Stroke Study. Arch Phys Med Rehabil 1995;76:399-405.

12. Kwakkel G, Wagenaar RC, Koelman TW, Lankhorst GJ, Koetsier JC. Effects of intensity of rehabilitation after stroke. Stroke 1997; 28:1550-1556.

13. Langhorne P, Wagenaar RC, Partridge C. Physiotherapy after stroke: more is better? Physiother Res Int 1996;1:75-88.

14. Kwakkel G, van Peppen R, Wagenaar RC, et al. Effects of augmented exercise therapy time after stroke: a meta-analysis. Stroke 2004;35:2529-2539.

15. Jette DU, Warren RL, Wirtalla C. The relation between therapy intensity and outcomes of rehabilitation in skilled nursing facilities. Arch Phys Med Rehabil 2005;86:373-379.

16. Bode RK, Heinemann AW, Semik P, Mallinson T. Relative importance of rehabilitation therapy characteristics on functional outcomes for persons with stroke. Stroke 2004;35:2537-2542.

17. Chen CC, Heinemann AW, Granger CV, Linn RT. Functional gains and therapy intensity during subacute rehabilitation: a study of 20 facilities. Arch Phys Med Rehabil 2002;83:1514-1523.

18. Humm JL, Kozlowski DA, James DC, Gotts JE, Schallert T. Use-dependent exacerbation of brain damage occurs during an early post-lesion vulnerable period. Brain Res 1998;783:286-292.

19. Dromerick AW, Edwards DF, Hahn M. Does the application of constraint-induced movement therapy during acute rehabilitation reduce hemiparesis after ischemic stroke? Stroke 2000;31:29842988.

20. Taub E, Pidikiti RD, DeLuca SC, Crago JE, Toole JF, Good DC. Effects of motor restriction of an unimpaired upper extremity and training on improving functional tasks and altering brain behaviors. In: Imaging in neurologic rehabilitation. New York: Demos; 1996:133-154.

21. Taub E, Miller NE, Novack TA, et al. Technique to improve chronic motor deficit after stroke. Arch Phys Med Rehabil 1993; 74:347-354.

22. Sterr A. Training-based interventions in motor rehabilitation after stroke: theoretical and clinical considerations. Behav Neurol 2004; 15:55-63.

23. van Peppen RPS, Kwakkel G, Wood-Dauphinee S, Hendriks HJM, van der Wees PJ, Dekker J. The impact of physical therapy on functional outcomes after stroke: what's the evidence? Clin Rehabil 2004;18:833-862.

24. Levine P, Page SJ. Modified constraint-induced therapy: a promising restorative outpatient therapy. Top Stroke Rehabil 2004;11: $1-10$.

25. Tarkka IM, Pitkanen K, Sivenius J. Paretic hand rehabilitation with constraint-induced movement therapy after stroke. Am J Phys Med Rehabil 2005;84:501-505.

26. Dettmers C, Teske U, Hamzei F, Uswatte G, Taub E, Weiller C. Distributed form of constraint-induced movement therapy improves functional outcome and quality of life after stroke. Arch Phys Med Rehabil 2005;86:204-209.

27. Kunkel A, Kopp B, Muller G, et al. Constraint induced movement therapy for motor recovery in chronic stroke patients. Arch Phys Med Rehabil 1999;80:624-628.

28. Miltner WHR, Bauder H, Sommer M, Dettmers C, Taub E. Effects of constraint-induced movement therapy on patients with chronic motor deficits after stroke: a replication. Stroke 1999;30:586-592.

29. Taub E, Uswatte G, King DK, Morris D, Crago JE, Chatterjee A. A placebo-controlled trial of constraint-induced movement therapy for upper extremity after stroke. Stroke 2006;37:1045-1049.

30. van der Lee JH. Constraint-induced movement therapy: some thoughts about theories and evidence. J Rehabil Med 2003:S41S45.

31. Siegert RJ, Lord S, Porter K. Constraint-induced movement therapy: time for a little restraint? Clin Rehabil 2004;18:110-114.

32. Dromerick AW. Evidence-based rehabilitation. The case for and against constraint-induced movement therapy. J Rehabil Res Dev 2003;40:vii-ix.

33. van der Lee JH, Wagenaar RC, Lankhorst GJ, Vogelaar TW, Deville WL, Bouter LM. Forced use of the upper extremity in chronic stroke patients. Stroke 1999;30:2369-2375.

34. Page SJ, Sisto S, Levine P, McGrath RE. Efficacy of modified constraint-induced movement therapy in chronic stroke: a singleblinded randomized controlled trial. Arch Phys Med Rehabil 2004; 85:14-18.

35. Parry RH, Lincoln NB, Vass CD. Effect of severity of arm impairment on response to additional physiotherapy early after stroke. Clin Rehabil 1999;13:187-198.

36. Hesse S, Schmidt H, Werner C, Bardeleben A. Upper and lower extremity robotic devices for rehabilitation and for studying motor control. Curr Opin Neurol 2003;16:705-710.

37. Krebs HI, Hogan N, Aisen ML, Volpe BT. Robot-aided neurorehabilitation. IEEE Trans Rehabil Eng 1998;6:75-87.

38. Volpe BT, Ferraro M, Lynch D, et al. Robotics and other devices in the treatment of patients recovering from stroke. Curr Atheroscler Rep 2004;6:314-319.

39. Ferraro M, Palazzolo JJ, Krol J, Krebs HI, Hogan N, Volpe BT. Robot-aided sensorimotor arm training improves outcome in patients with chronic stroke. Neurology 2003;61:1604-1607.

40. Fasoli SE, Krebs HI, Stein J, Frontera WR, Hogan N. Effects of robotic therapy on motor impairment and recovery in chronic stroke. Arch Phys Med Rehabil 2003;84:477-482.

41. Reinkensmeyer DJ, Dewald JP, Rymer WZ. Guidance-based quantification of arm impairment following brain injury: a pilot study. IEEE Trans Rehabil Eng 1999;7:1-11.

42. Kahn LE, Lum PS, Rymer WZ, Reinkensmeyer DJ. Robot-assisted movement training for the stroke-impaired arm: does it matter what the robot does? J Rehabil Res Dev (in press).

43. Lum PS, Burgar CG, Shor PC, Majmundar M, Van der Loos M. Robot-assisted movement training compared with conventional therapy techniques for the rehabilitation of upper-limb motor function after stroke. Arch Phys Med Rehabil 2002;83:952-959.

44. Lum PS, Burgar CG, Shor PC. Evidence for improved muscle activation patterns after retraining of reaching movements with the MIME robotic system in subjects with post-stroke hemiparesis. IEEE Trans Neural Syst Rehabil Eng 2004;12:186-194.

45. Lindeman E, Spaans F, Reulen J, Leffers P, Drukker J. Progressive resistance training in neuromuscular patients. Effects on force and surface EMG. J Electromyogr Kinesiol 1999;9:379-384.

46. McCartney N, Moroz D, Garner SH, McComas AJ. The effects of strength training in patients with selected neuromuscular disorders. Med Sci Sports Exerc 1988;20:362-368. 
47. Duchateau J, Enoka RM. Neural adaptations with chronic activity patterns in able-bodied humans. Am J Phys Med Rehabil 2002;81: S17-S27.

48. Sharp SA, Brouwer BJ. Isokinetic strength training of the hemiparetic knee: effects on function and spasticity. Arch Phys Med Rehabil 1997;78:1231-1236.

49. Ouellette MM, LeBrasseur NK, Bean JF, et al. High-intensity resistance training improves muscle strength, self-reported function, and disability in long-term stroke survivors. Stroke 2004;35: 1404-1409.

50. Lum PS, Burgar CG, Shor PC. Evidence for strength imbalances as a significant contributor to abnormal synergies in hemiparetic subjects. Muscle Nerve 2003;27:211-221.

51. Morrissey MC, Harman EA, Johnson MJ. Resistance training modes: specificity and effectiveness. Med Sci Sports Exerc 1995; 27:648-660.

52. Jones DA, Rutherford OM. Human muscle strength training: the effects of three different regimens and the nature of the resultant changes. J Physiol 1987;391:1-11.

53. Barbeau H, Wainberg M, Finch L. Description and application of a system for locomotor rehabilitation. Med Biol Eng Comput 1987; 25:341-344.

54. Edgerton VR, de Guzman CP, Gregor RJ, Roy RR, Hodgson JA, Lovely RG. Trainability of the spinal cord to generate hindlimb stepping patterns in adult spinalized cats. In: Shimamura SM, Grillner S, Edgerton VR, eds. Neurobiological basis of human locomotion. Tokyo: Japan Scientific Societies Press; 1991;411423.

55. Belanger M, Drew T, Provencher J, Rossignol S. A comparison of treadmill locomotion in adult cats before and after spinal transection. J Neurophysiol 1996;76:471-491.

56. Barbeau H, Rossignol S. Recovery of locomotion after chronic spinalization in the adult cat. Brain Res 1987;412:84-95.

57. Lovely RG, Gregor RJ, Roy RR, Edgerton VR. Effects of training on the recovery of full-weight-bearing stepping in the adult spinal cat. Exp Neurol 1986;92:421-435.

58. de Leon RD, Hodgson JA, Roy RR, Edgerton VR. Locomotor capacity attributable to step training versus spontaneous recovery after spinalization in adult cats. J Neurophysiol 1998;79:13291340 .

59. Wolpaw JR, Tennissen AM. Activity-dependent spinal cord plasticity in health and disease. Annu Rev Neurosci 2001;24:807-843.

60. van de Crommert HWAA, Mulder T, Duysens J. Neural control of locomotion: sensory control of the central patter generator and its relation to treadmill training. Gait Posture 1998;7:251-263.

61. de Leon RD, Roy RR, Edgerton VR. Is the recovery of stepping following spinal cord injury mediated by modifying existing neural pathways or by generating new pathways? A perspective. Phys Ther 2001;81:1904-1911.

62. Behrman AL, Harkema SJ. Locomotor training after human spinal cord injury: a series of case studies. Phys Ther 2000;80:688-700.

63. Wernig A, Muller S. Laufband locomotion with body weight support improved walking in persons with severe spinal cord injuries. Paraplegia 1992;30:229-238.

64. Wernig A, Nanassy A, Muller S. Laufband (treadmill) therapy in incomplete paraplegia and tetraplegia. J Neurotrauma 1999;16: $719-726$.

65. Dietz V. Locomotor training in paraplegic patients. Ann Neurol 1995;386:965.

66. Hesse S, Bertelt C, Jahnke MT, et al. Treadmill training with partial body weight support compared with physiotherapy in nonambulatory hemiparetic stroke patients. Stroke 1995;26:976981.

67. Mauritz KH, Hesse S, Platz T. Late recovery of motor functions. Adv Neurol 1997;73:395-408.
68. Visintin M, Barbeau H, Korner-Bitensky N, Mayo NE. A new approach to retrain gait in stroke patients through body weight support and treadmill stimulation. Stroke 1998;29:1122-1128.

69. Dobkin BH, Apple D, Barbeau H, et al. Methods for a randomized trial of weight-supported treadmill training versus conventional training for walking during inpatient rehabilitation after incomplete traumatic spinal cord injury. Neurorehabil Neural Repair 2003;17:153-167.

70. Dobkin B, Apple D, Barbeau H, et al. Weight-supported treadmill vs over-ground training for walking after acute incomplete SCI. Neurology 2006;66:484-493.

71. Di Giovanna I, Hayes G. The drug development process. In: Principles of clinical research. Petersfield, United Kingdom: Wrightson Biomedical; 2001:1-16.

72. Knapp HD, Taub E, Berman AJ. Movements in monkeys with deafferented forelimbs. Exp Neurol 1963;7:305-315.

73. Schallert T, Leasure JL, Kolb B. Experience-associated structural events, subependymal cellular proliferative activity, and functional recovery after injury to the central nervous system. J Cereb Blood Flow Metab 2000;20:1513-1528.

74. Edgerton VR, Tillakaratne NJK, Bigbee AJ, de Leon RD, Roy RR Plasticity of the spinal neural circuitry after injury. Annu Rev Neurosci 2004;27:145-167.

75. Richard Green A, Odergren T, Ashwood T. Animal models of stroke: do they have value for discovering neuroprotective agents? Trends Pharmacol Sci 2003;24:402-408.

76. Dobkin BH, Havton LA. Basic advances and new avenues in therapy of spinal cord injury. Annu Rev Med 2004;55:255-282.

77. Dromerick AW, Morris LB, eds. Acute ischemic stroke lesions in humans: how do they compare to animal models used to study stroke recovery? Washington, DC; Society for Neuroscience 2005; Abstract. 2005.

78. Page SJ, Levine P, Leonard AC. Modified constraint-induced therapy in acute stroke: a randomized controlled pilot study. Neurorehabil Neural Repair 2005;19:27-32.

79. McDonald JW, Becker D, Sadowsky CL, Jane JA Sr, Conturo TE, Schultz LM. Late recovery following spinal cord injury. Case report and review of the literature. J Neurosurg 2002;97:S252S265.

80. Ricamato AL, Hidler JM. Quantification of the dynamic properties of EMG patterns during gait. J Electromyogr Kinesiol 2005;15: 384-92.

81. Gresham GE. Stroke outcome research. Stroke 1986;17:358-360.

82. Wylie CM. The value of early rehabilitation in stroke. Geriatrics 1970;25:107-113.

83. Ottenbacher KJ, Jannell S. The results of clinical trials in stroke rehabilitation research. Arch Neurol 1993;50:37-44.

84. Paolucci S, Antonucci G, Grasso MG, et al. Early versus delayed inpatient stroke rehabilitation: a matched comparison conducted in Italy. Arch Phys Med Rehabil 2000;81:695-700.

85. Jones TA, Schallert T. Use-dependent growth of pyramidal neurons after neocortical damage. J Neurosci 1994;14:2140-2152.

86. Kempermann G, Gage FH. Experience-dependent regulation of adult hippocampal neurogenesis: effects of long-term stimulation and stimulus withdrawal. Hippocampus 1999;9:321-332.

87. Biernaskie J, Chernenko G, Corbett D. Efficacy of rehabilitative experience declines with time after focal ischemic brain injury. J Neurosci 2004;24:1245-1254.

88. Barbeau H, Visintin M. Optimal outcomes obtained with bodyweight support combined with treadmill training in stroke subjects. Arch Phys Med Rehabil 2003;84:1458-1465.

89. Dodds TA, Martin DP, Stolov WC, and Deyo RA. A validation of the functional independence measure and its performance among rehabilitation in patients. Arch Phys Med Rehabil 1993;74:531536. 